\title{
A Comparison of Robust Criteria for Vehicle Routing Problem with Soft Time Windows
}

\author{
Thanasak Mouktonglang $\mathbb{1 D}^{1,2}$ and Phannipa Worapun $\left.{ }^{3}\right)^{3}$ \\ ${ }^{1}$ Center of Excellence in Mathematics and Applied Mathematics, Department of Mathematics, Faculty of Science, \\ Chiang Mai University, Chiang Mai 50200, Thailand \\ ${ }^{2}$ Thailand or Centre of Excellence in Mathematics, CHE, Si Ayutthaya Rd., Bangkok 10400, Thailand \\ ${ }^{3}$ Department of Applied Mathematics and Statistics, Rajamangala University of Technology Isan, Nakhon \\ Ratchasima 30000, Thailand
}

Correspondence should be addressed to Phannipa Worapun; phannipa.math@gmail.com

Received 5 July 2019; Revised 30 September 2019; Accepted 8 October 2019; Published 23 October 2019

Academic Editor: Tamer Eren

Copyright (c) 2019 Thanasak Mouktonglang and Phannipa Worapun. This is an open access article distributed under the Creative Commons Attribution License, which permits unrestricted use, distribution, and reproduction in any medium, provided the original work is properly cited.

In this study, we focus on robust criteria for vehicle routing problems with soft time windows (VRPSTW). The main objective is to find a robust solution that provides the best for the worst case performance for VRPSTW under uncertain travel times. The robust criteria are used in this study such as absolute robustness, robust deviation, and relative robustness as a basis for comparison. The VRPSTW becomes complex when the travel times are uncertain. This uncertainty can be caused by traffic jams, accidents, or inclement weather conditions. The experiment uses benchmarking problems. The number of scenarios is generated randomly into intervals of travel time, equal to 4, 6, and 8 instances for each problem set. Each set of problem instances can be denoted by the percentage of uncertainty $\alpha$, equal to $0.2,0.4,0.6$, and 0.8 . This study will demonstrate that the most indicated robust criteria for these situations are robust deviation and relative robustness. The most important part of the decision maker is to determine the uncertainty percentage to cover all uncertainties that need to be considered.

\section{Introduction}

The vehicle routing problem (VRP) is in the field of transportation and logistics. It plays an important role for driving the smart city. The logistic for smart city should support all logistic platforms and help users to make important decisions. The problem of providing transportation routes from a central depot to customers in order to save transportation costs is presented by Dantzig and Ramser [1]. The vehicle routing problem with soft time windows (VRPSTW) is a variant of the VRP. The solution of the VRPSTW is a set of routes consisting of sequences of customers. Each route is designed to insure delivery within specified time frames called windows. Moreover, in VRPSTW, the time windows can be adjusted if a penalty is paid. Therefore, the objective of the VRPSTW problem is to find transportation routes to save the travel costs and penalty costs of transportation. The service time interval in
VRPSTW is shown in Figure 1. Kabcome and Mouktonglang [2] presented an explicitly written mathematical model for VRPSTW and the problem was solved efficiently. From the numerical result, it was clear that by adding the service penalty constraints, the problem could be solved sufficiently and it provided another solution to the problem. Taillard et al. [3] used tabu search, metaheuristic approach, for the vehicle routing problem with soft time windows. Min [4] solved a multiobjective vehicle routing problem with soft time windows for the public library distribution system.

This VRPSTW becomes complex when the travel times are uncertain due to traffic conditions such as traffic jams, accidents, or bad weather conditions. Agra et al. [5] considered a robust counterpart for the VRP. They consider the problem that arises in maritime transportation where delays should be taken into account. Therefore, we are interested in studying the VRPSTW problem with uncertain travel time. The general principles used for solving a problem with 


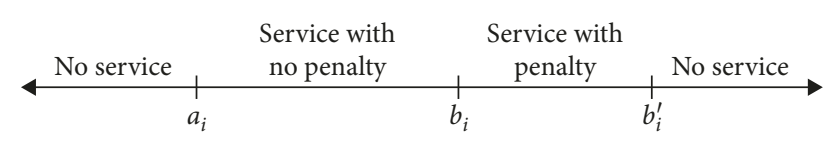

FIgURE 1: Service time interval in VRPSTW.

uncertainties are divided into two types: the deterministic optimization approach and the stochastic optimization approach. The deterministic optimization approach has some good properties such as the mathematical models are so much easier to write and to understand. The size of the deterministic optimization problem is usually smaller than the stochastic ones. However, it also has some disadvantages when the problem contains uncertainties because this method finds solutions by estimating uncertainty to some value or by using the most frequent situation to represent the problem. The solution is to solve only the selected situation. When the uncertainty is higher, the efficiency of this method is immediately decreased. The stochastic optimization approach is to find solutions from more than one uncertainty estimation. See [6] for more details where the authors considered stochastic version for VRP with time windows. This method requires historical uncertainty data that are analyzed using statistical methods and expressed in terms of probability values to represent uncertainty. In this way, the stochastic method has the ability to resolve several problems containing uncertainty. However, if we consider the actual application, the stochastic method presents difficulties and does not respond to the needs of the decision maker because data collection requires a long period of time. The major disadvantage of the stochastic method is that the solution can only be a solution that comes from the expected value. In this situation, the decision maker must assume a risk when the actual situation occurs at the minimum or maximum range. Even if this situation occurs only once, it may have serious consequences in terms of reliability or lost opportunity.

This study focuses on responding to the actual use of decision makers by offering a robust optimization approach. The solutions must be applicable to different conditions of use. Robustness approach is an important objective of this research, which is to find the most suitable route that is able to withstand the uncertainty of travel time. This paper proposes an approach to the robust discrete optimization of VRPSTW with uncertain travel times. By solving the VRPSTW multiple times, the minimax optimization problem becomes the main objective of this paper (i.e., to find a robust solution under robust criteria).

In the following section, we provide a review of relevant literature in robust discrete optimization problems. Section 3 presents the method of solving the problems. Section 4 represents the results and related discussion. The numerical experiments use benchmark data from the VRP problem in Solomon's VRPTW [7]. Finally, the conclusions are presented in Section 5.

\section{Literature Review}

The problem of decision-making under this uncertainty began to be discussed in 1955 by Dantzig [8] and was developed by Charnes and Cooper [9]. A lot of studies on uncertainty rely on stochastic programming techniques based on either recourse models or probabilistic constraints with uncertainty in demands, supplies, travel times, and many others. We refer to Ben-Tal et al. [10] where these two approaches are discussed in detail. In this study, the authors are interested in finding solutions that satisfy conditions to withstand uncertainty or finding the best value in a worst case scenario. Kouvelis and $\mathrm{Yu}$ [11] described a robust decision-making framework which can be used to find durable solutions that consist of 3 main parts: (1) creating uncertain data with various situations, (2) selecting criteria for judging durable solutions, and (3) creating the most suitable model for durable decisions.

The process for finding solutions that are resistant to uncertainty is as follows: let $S$ be a set of scenarios, $s \in S, D$ be data sets, $D_{s}$ be a set of data that are uncertain according to the situation $s, F_{s}$ be the set of all possible solutions for each $s$ and $F_{s} \neq \varnothing, X$ be the set of decision variables and $X \in F_{s}$, and $f\left(X, D_{s}\right)$ be the function used for finding solution $X$ of each scenario $D_{s}$ and $X_{s}^{*}$ be the optimal solution of each scenario $D_{s}$ satisfying the following problem:

$$
Z_{S}=f\left(X_{s}^{*}, D_{s}\right)=\min _{X \in F_{s}} f\left(X, D_{s}\right) .
$$

From equation (1), it is known that the number to solve deterministic problems is equal to the number of situations. We used three robust criteria for finding the robust solution and are given as follows.

Definition 1. The absolute robustness criterion $Z_{A}$ : let $X_{A}$ be the decision variable of absolute robustness criterion determined by

$$
Z_{A}=\max _{s \in S} f\left(X_{A}, D_{s}\right)=\min _{X \in \cap_{s \in S} F_{s}} \max _{s \in S} f\left(X, D_{s}\right)
$$

Definition 2. The robust deviation $Z_{D}$ : let $X_{D}$ be the decision variable of robust deviation criterion determined by

$$
\begin{aligned}
Z_{D} & =\max _{s \in S}\left(f\left(X_{D}, D_{s}\right)-f\left(X_{s}^{*}, D_{s}\right)\right) \\
& =\min _{X \in \cap_{s \in S} F_{s}} \max _{s \in S}\left(f\left(X, D_{s}\right)-f\left(X_{s}^{*}, D_{s}\right)\right) .
\end{aligned}
$$

Definition 3. The relative robustness $Z_{R}$ : let $X_{R}$ be the decision variable of robust deviation criterion determined by

$$
\begin{aligned}
Z_{R} & =\max _{s \in S} \frac{f\left(X_{R}, D_{s}\right)-f\left(X_{s}^{*}, D_{s}\right)}{f\left(X_{s}^{*}, D_{s}\right)} \\
& =\min _{X \in \cap_{s \in S} F_{s}} \max _{s \in S} \frac{f\left(X, D_{s}\right)-f\left(X_{s}^{*}, D_{s}\right)}{f\left(X_{s}^{*}, D_{s}\right)} .
\end{aligned}
$$

\section{Methodology}

This section presents the mathematical model for solving the robust solution, that is, the best of the worst case 
performance for VRPSTW under uncertain travel times. The first part presents the vehicle routing problems with soft time windows (VRPSTW). The second part presents the robust vehicle routing with soft time windows (RVRPSTW).

3.1. The Vehicle Routing Problems with Soft Time Windows (VRPSTW). The VRPSTW is defined on a directed graph $G=(N, A)$, where $N=\{o, d, 1,2,3, \ldots, n\}$ is a set of vertices $(o, d$ is the depot) and $\mathrm{A}$ is a set of $\operatorname{arcs}(i, j)$ representing the connections between the depot and customers or among the customers $A=\{(i, j) \mid i, j \in N, i \neq j\}$.

The following notations are introduced to formulate the mathematical model of VRPSTW.

\subsubsection{Indices}

$i, j, n$ index of nodes

$k$ index of vehicle

\subsubsection{Sets}

$N=\{o, d, 1,2,3, \ldots, n\}$ be a node set (o is origin and $d$ is destination)

$N^{*}=N-\{o, d\}$ be a set of customers

$K=\{1,2,3, \ldots, k\}$ be a set of vehicles

\subsubsection{Decision Variables}

$x_{k, i, j}= \begin{cases}1 ; & \text { if vehicle } k \text { travels directly from node } i \text { to } j ; \\ 0 ; & \text { otherwise }\end{cases}$

$y_{i}$ is the violation degree of the upper bound of soft time windows at the customer $i$

$w_{i}$ be the arrival time at the customer $i$

\subsubsection{Input Parameters}

$c_{i, j}$ be the travel cost from $i$ to $j$

$t_{i, j}$ be a time from node $i$ to $j$

$x c_{i}$ be the coordinate $x$ of $i$

$y c_{i}$ be the coordinate $y$ of $i$,

$d s_{i, j}$ be a distance between customers $i$ and $j$

$d s_{i, j}=\sqrt{\left(x c_{i}-x c_{j}\right)^{2}+\left(y c_{i}-y c_{j}\right)^{2}}$

$d_{i}$ be the demand of the customer $i$, and $p_{i}$ be penalty cost per hour for the lateness of customer $i$

$a_{i}$ be the early time to service the node $i$ without penalty $b_{i}$ be the last time to service the note $i$ without penalty $b_{i}^{\prime}$ be the last time to service the node $i$ with penalty $v_{i}$ be a service time at the customer $i$ $q_{k}$ be the capacity of the vehicle $k$

The solution to the VRPSTW must satisfy the following:

(1) Every vehicle starts and finishes only at the central depot

(2) Every customer can be served by one and only one vehicle
(3) The total demand must not exceed the capacity of the vehicle

(4) Each customer must be served in the related hard time window

(5) If the vehicle arrives early at the customer $i$, it can wait until the time instant to start service

The VRPSTW can then be stated mathematically as follows:

$$
\operatorname{Min}\left(\sum_{k, i, j} c_{i, j} d s_{i, j} x_{k, i, j}+\sum_{i} p_{i} y_{i}\right),
$$

subject to

$$
\begin{aligned}
& \sum_{j} x_{k, j, i}-\sum_{j} x_{k, i, j}= \begin{cases}1 ; & i=d, \\
-1 ; & i=o, \\
0 ; & \text { otherwise, }\end{cases} \\
& \forall i \in N, \forall k \in K \text {, } \\
& \sum_{k, j} x_{k, i, j}=1 ; \quad \forall i \in N^{*}, \\
& x_{k, i, i}=0 ; \quad \forall k \in K, \forall i \in N, \\
& \sum_{i \in N^{*}}\left(d_{i} \sum_{j \in N} x_{k, i, j}\right) \leq q_{k} ; \quad \forall k \in K, \\
& w_{i}+t_{i, j}+v_{i}-w_{j} \leq\left(1-x_{k, i, j}\right) \max \left(b_{i}^{\prime}+t_{i, j}+v_{i}-a_{j}, 0\right) ; \\
& \forall i, j \in N \text {, } \\
& a_{i} \leq w_{i} \leq b_{i}^{\prime} ; \quad \forall i \in N, \\
& y_{i}=\max \left(w_{i}-b_{i}, 0\right) ; \quad \forall i \in N^{*} .
\end{aligned}
$$

We minimize the objective function (5) which consists of two components: the first component represents travel cost and the second component is the total penalties of the violation of soft time windows.

The constraints (6) are the flow conservation of a vehicle. That is, for $i=d$, every vehicle ends only once at the depot and no arc originates from node $d$; for $i=o$, every vehicle starts only once at the depot and no arc terminates at node $o$; and $i \in N^{*}$ indicates that exactly one vehicle enters and leaves each customer node.

The constraints (7) require that each customer is serviced exactly once.

The constraints (8) are the flow conservation constraints which describe the vehicle path.

The constraints (9) ensure that the vehicle capacity constraint is not violated.

The constraints (10) ensure feasibility of the time schedule.

The constraints (11) assure that the starting time of services does not violate the given hard time windows. 
The constraints (12) are the violation of soft time windows.

3.2. The Robust Vehicle Routing with Soft Time Windows (RVRPSTW). The approach for solving the robust discrete optimization VRPSTW with uncertain travel times is equivalent to the minimax optimization problem. We used a robust decision-making framework of Kouvelis and Yu [11] to find durable solutions. First, the uncertainty data are created by considering situations of travel time between depot and customers and the time to travel between each customer. Indeed, let

$S$ be the set of all possible scenarios

$t$ be the travel time at base case scenario

$t_{s}$ be the travel time of each scenario $s$ dependent on $\alpha$ such as $0 \leq \alpha \leq 1$, and $t_{s}$ selected randomly from $t_{s} \in((1-\alpha) t,(1+\alpha) t)$

$F_{s}$ be the set of all possible solutions at each scenario $s$, and $\cap_{s \in S} F_{s} \neq \varnothing$

$X$ be the decision variable and $X \in F_{s}$

$X_{s}^{*}$ be the optimal solution at each scenario $s$

$f\left(X, t_{s}\right)$ is the function used for finding solution $X$ of each scenario

$Z_{s}$ be the optimal value at each scenario from $X_{s}^{*}$

So that, for each situation, we must find the optimal value $Z_{s}$ from the aforementioned VRPSTW problem:

$$
Z_{s}=f\left(X_{s}^{*}, t_{s}\right)=\min _{X \in F_{s}} f\left(X, t_{s}\right) .
$$

Therefore, we can find the optimal value of each scenario by using equation (13). Notice, we solve problem (13) as many times as the number of scenarios. Then, equation (13) is a function to represent the robust counterpart.

After we have the optimal value of VRPSTW for each scenario, the next step is to find the main goal of the minimax optimization problem, which is to find the robust optimal value $Z^{*}$.

We start by finding the highest value $h_{x}(S)$ of all scenarios. The robust criteria that are considered are absolute robustness $Z_{A}$ as described in Definition 1, robust deviation $Z_{D}$ as described in Definition 2, and relative robustness $Z_{R}$ as described in Definition 3.

After that, we select the robust solution from the results from all possible solutions that provide the lowest value that can be obtained in the event of the worst case.

The robust vehicle routing with soft time windows (RVRPSTW) can then be stated as follows:

$$
Z^{*}=\min \left\{h_{X}(S) \mid g_{s}(X) \leq h_{X}(S) ; s \in S, X \in \cap_{s \in S} F_{s}\right\},
$$

subject to

$$
g_{s}(X)= \begin{cases}f\left(X, t_{s}\right), & \text { for absolute robustness, } \\ f\left(X, t_{s}\right)-Z_{s}, & \text { for robust deviation, } \\ \frac{f\left(X, t_{s}\right)-Z_{s}}{Z_{s}}, & \text { for relative robustness, }\end{cases}
$$

where $f\left(X, t_{s}\right)$ is the function used for finding solution $X$ of each scenario and $Z_{s}$ is the optimal value of VRPSTW for each scenario.

Finally, we compared the performance of the three criteria by using the relative ratio:

$$
P=\frac{Z^{*}-Z}{Z}
$$

where $Z^{*}$ is the robust solution $Z_{A}$ or $Z_{D}$ or $Z_{R}$ and $Z$ is the solution of base case.

The process to find RVRPSTW is summarized as follows:

(1) Construct a base case scenario by fixed travel time $t$.

(2) Generate all possible scenarios by percentage of uncertainty $0 \leq \alpha \leq 1$ by selecting $t_{s}$ from $((1-\alpha) t,(1+\alpha) t)$.

(3) Find optimal value $Z_{s}$ and optimal solution $X_{s}$ of each scenario $s$.

(4) Finding the highest value $h_{x}(S)$ of all scenarios with the robust criteria:

$$
g_{s}(X)= \begin{cases}f\left(X, t_{s}\right), & \text { for absolute robustness, } \\ f\left(X, t_{s}\right)-Z_{s}, & \text { for robust deviation, } \\ \frac{f\left(X, t_{s}\right)-Z_{s}}{Z_{s}}, & \text { for relative robustness. }\end{cases}
$$

(5) Find $X^{*}$ which is the robust solution from

$$
Z^{*}=\min \left\{h_{X}(S) \mid g_{s}(X) \leq h_{X}(S) ; s \in S, X \in \cap_{s \in S} F_{s}\right\} \text {. }
$$

(6) Comparing the performance of the three criteria by using the relative ratio $P=\left(Z^{*}-Z\right) / Z$.

\section{Results and Discussion}

This demonstration uses benchmarking problem of Solomon's VRPTW [7]. We randomly generate soft time windows in VRPSTW from the time frames of VRPTW. Solomon's VRPSTW divides the problem into 6 types of instances, including type R1, R2, C1, C2, RC1, and RC2. In this study, we find the robust solution of R101, C101, RC101, R201, C201, and RC201 with the number of customers equal to 25. The number of scenarios is generated randomly into intervals of travel time, equal to 4, 6, and 8 instances for each problem set. Each set of problem instances can be denoted by the percentage of uncertainty $\alpha$, equal to $0.2,0.4,0.6$, and 0.8 .

We examine the robust solution of case R101 by using 6 as the number of scenarios and 0.4 as $\alpha$ in order to observe the behavior of the robust solution. We import data from Solomon's VRPSTW problem, and let $c_{i, j}=1, p_{i}=1$, and $b_{i}^{\prime}=b_{i}+20$. From these imported data, we have coded and solved VRPSTW by using AIMMS 4.9. The results are given in Tables 1-4.

Next, we present the minimax optimization problem, which is to find the robust optimal value.

From the minimax optimization problem shown in Tables 2-4, we can conclude the process and show the results in Table 5. 
TABLE 1: The optimal value of each scenario.

\begin{tabular}{cccccccr}
\hline & Base case & $s_{1}$ & $s_{2}$ & $s_{3}$ & $s_{4}$ & $s_{5}$ & $s_{6}$ \\
\hline$Z_{s}$ & 608.832 & 590.996 & 601.373 & 614.277 & 620.412 & 589.587 & 596.467 \\
\hline
\end{tabular}

TABLE 2: The absolute robustness $Z_{A}$.

\begin{tabular}{|c|c|c|c|c|c|c|c|}
\hline \multirow{2}{*}{ Solutions } & \multicolumn{6}{|c|}{ Scenarios } & \multirow{2}{*}{$h_{X_{i}^{*}}(S)$} \\
\hline & $g_{s_{1}}\left(X_{i}^{*}\right)$ & $g_{s_{2}}\left(X_{i}^{*}\right)$ & $g_{s_{3}}\left(X_{i}^{*}\right)$ & $g_{s_{4}}\left(X_{i}^{*}\right)$ & $g_{s_{5}}\left(X_{i}^{*}\right)$ & $g_{s_{6}}\left(X_{i}^{*}\right)$ & \\
\hline$X_{1}^{*}$ & 590.996 & 602.278 & 617.482 & 635.623 & 589.587 & 597.210 & 635.623 \\
\hline$X_{2}^{*}$ & 592.897 & 601.373 & 618.865 & 643.133 & 592.149 & 596.467 & 643.133 \\
\hline$X_{3}^{*}$ & 598.923 & 604.639 & 614.277 & 625.051 & 598.443 & 601.427 & 625.051 \\
\hline$X_{4}^{*}$ & 618.330 & 618.330 & 618.330 & 620.412 & 618.330 & 618.330 & 620.412 \\
\hline$X_{5}^{*}$ & 590.996 & 602.278 & 617.482 & 635.623 & 589.587 & 597.210 & 635.623 \\
\hline \multirow[t]{2}{*}{$X_{6}^{*}$} & 592.897 & 601.373 & 618.865 & 643.133 & 592.149 & 596.467 & 643.133 \\
\hline & & & & & & $Z_{A}=$ & 620.412 \\
\hline
\end{tabular}

TABLE 3: The robust deviation $Z_{D}$.

\begin{tabular}{lcccccc}
\hline \multirow{2}{*}{ Solutions } & \multicolumn{5}{c}{ Scenarios } \\
& $g_{s_{1}}\left(X_{i}^{*}\right)$ & $g_{s_{2}}\left(X_{i}^{*}\right)$ & $g_{s_{3}}\left(X_{i}^{*}\right)$ & $g_{s_{1}}\left(X_{i}^{*}\right)$ & $g_{s_{2}}\left(X_{i}^{*}\right)$ & $g_{s_{6}}\left(X_{i}^{*}\right)$ \\
\hline$X_{1}^{*}$ & 0 & 0.9054 & 3.2054 & 15.2109 & 0 & 0.7432 \\
$X_{2}^{*}$ & 1.9012 & 0 & 4.5882 & 22.7209 & 2.5623 & 0 \\
$X_{3}^{*}$ & 7.9277 & 3.2665 & 0 & 4.6388 & 8.8555 & 4.9598 \\
$X_{4}^{*}$ & 27.3343 & 16.9572 & 4.0530 & 0 & 22.7209 \\
$X_{5}^{*}$ & 0 & 0.9054 & 3.2054 & 15.2109 & 0 & $\mathbf{8 . 8 5 5 5}$ \\
$X_{6}^{*}$ & 1.9012 & 0 & 4.5882 & 22.7209 & 2.5623 & 0.7432 \\
& & & & & & 0 \\
\hline
\end{tabular}

TABLE 4: The relative robustness $Z_{R}$.

\begin{tabular}{lcccccc}
\hline \multirow{2}{*}{ Solutions } & & \multicolumn{2}{c}{ Scenarios } & \\
& $g_{s_{1}}\left(X_{i}^{*}\right)$ & $g_{s_{2}}\left(X_{i}^{*}\right)$ & $g_{s_{3}}\left(X_{i}^{*}\right)$ & $g_{s_{4}}\left(X_{i}^{*}\right)$ & $g_{s_{5}}\left(X_{i}^{*}\right)$ & $g_{s_{6}}\left(X_{i}^{*}\right)$ \\
\hline$X_{1}^{*}$ & 0 & 0.0015 & 0.0052 & 0.0245 & 0 & 0.0012 \\
$X_{2}^{*}$ & 0.0032 & 0 & 0.0075 & 0.0366 & 0.0043 & 0 \\
$X_{3}^{*}$ & 0.0134 & 0.0054 & 0 & 0.0075 & 0.0150 & 0.0083 \\
$X_{4}^{*}$ & 0.0463 & 0.0282 & 0.0066 & 0 & 0.04875 & 0.0367 \\
$X_{5}^{*}$ & 0 & 0.0015 & 0.0052 & 0.0245 & 0 & 0.0366 \\
$X_{6}^{*}$ & 0.00327 & 0 & 0.0075 & 0.0366 & 0.0043 & 0.0150 \\
& & & & & 0.0488 \\
\hline
\end{tabular}

TABLE 5: The result of RVRPSTW.

\begin{tabular}{lcccc}
\hline Criteria & Robust optimal value & Robust optimal solution & Cost & Relative ratio \\
\hline$Z_{A}$ & 620.4120246 & $X_{4}^{*}$ & 620.4120246 & 0.018665753 \\
$Z_{D}$ & 8.8554838 & $X_{3}^{*}$ & 598.4425665 & -0.017063834 \\
$Z_{R}$ & 0.015019806 & $X_{3}^{*}$ & 598.4425665 & -0.017360063 \\
\hline
\end{tabular}

TABLE 6: The optimal value of base case problem for each percentage of uncertainty $\alpha$.

\begin{tabular}{lcccc}
\hline$\alpha$ & 0.2 & 0.4 & 0.6 & 0.8 \\
Optimal value & 597.6814133 & 608.8315627 & 618.3299155 & 621.316885 \\
\hline
\end{tabular}

In addition, we consider the cost for the robust solution of the problem R101 when the number of scenarios is 4,6 , and 8 . The percentage of uncertainty $\alpha$ is equal to $0.2,0.4,0.6$, and 0.8 . The results are given in Tables 6-8.

In the comparison between solutions from the robust criteria, we found that the important parameter that affects the solutions is the uncertainty percentage $\alpha$. The results in Table 6 show that when the uncertainty percentage $\alpha$ increases from 0.2 to 0.8 , the optimal values are also increased. This is because the travel time in each route is determined by the minimum and maximum time periods. When the value of $\alpha$ increases, the ranges of times are wider, while the 
TABLE 7: The solution of RVRPSTW for R101.

\begin{tabular}{|c|c|c|c|c|}
\hline & \multirow{2}{*}{ Scenario } & \multicolumn{3}{|c|}{ Cost } \\
\hline & & $Z_{A}$ & $Z_{D}$ & $Z_{R}$ \\
\hline \multirow{4}{*}{$|S|=4$} & $\alpha=0.2$ & 608.7279786 & 610.4065438 & 610.4065438 \\
\hline & $\alpha=0.4$ & 620.4120246 & 598.9233066 & 598.9233066 \\
\hline & $\alpha=0.6$ & 637.7034653 & 618.3299155 & 618.3299155 \\
\hline & $\alpha=0.8$ & 672.1878165 & 628.1196809 & 628.1196809 \\
\hline \multirow{4}{*}{$|S|=6$} & $\alpha=0.2$ & 609.8976676 & 611.9471293 & 611.9471293 \\
\hline & $\alpha=0.4$ & 620.4120246 & 598.4425665 & 598.4425665 \\
\hline & $\alpha=0.6$ & 637.7034653 & 618.3299155 & 618.3299155 \\
\hline & $\alpha=0.8$ & 672.1878165 & 628.1196809 & 628.1196809 \\
\hline \multirow{4}{*}{$|S|=8$} & $\alpha=0.2$ & 609.8976676 & 611.9471293 & 611.9471293 \\
\hline & $\alpha=0.4$ & 621.0159409 & 598.4425665 & 598.4425665 \\
\hline & $\alpha=0.6$ & 637.7034653 & 618.3299155 & 618.3299155 \\
\hline & $\alpha=0.8$ & 672.1878165 & 628.1196809 & 628.1196809 \\
\hline
\end{tabular}

TABle 8: The ratio $P$ of performance for robust criteria.

\begin{tabular}{|c|c|c|c|c|}
\hline \multicolumn{2}{|c|}{ Scenario } & \multicolumn{3}{|c|}{ Relative ratio } \\
\hline & & $Z_{A}$ & $Z_{D}$ & $Z_{R}$ \\
\hline \multirow{4}{*}{$|S|=4$} & $\alpha=0.2$ & 0.018482364 & 0.021290825 & 0.021290825 \\
\hline & $\alpha=0.4$ & 0.019020798 & -0.016274216 & -0.016274216 \\
\hline & $\alpha=0.6$ & 0.03133206 & 0 & 0 \\
\hline & $\alpha=0.8$ & 0.081875984 & 0.010948996 & 0.010948996 \\
\hline \multirow{4}{*}{$|S|=6$} & $\alpha=0.2$ & 0.020439408 & 0.023868428 & 0.023868428 \\
\hline & $\alpha=0.4$ & 0.019020798 & -0.017063827 & -0.017063827 \\
\hline & $\alpha=0.6$ & 0.03133206 & 0 & 0 \\
\hline & $\alpha=0.8$ & 0.081875984 & 0.010948996 & 0.010948996 \\
\hline \multirow{4}{*}{$|S|=8$} & $\alpha=0.2$ & 0.020439408 & 0.023868428 & 0.023868428 \\
\hline & $\alpha=0.4$ & 0.020012724 & -0.017063827 & -0.017063827 \\
\hline & $\alpha=0.6$ & 0.03133206 & 0 & 0 \\
\hline & $\alpha=0.8$ & 0.081875984 & 0.010948996 & 0.010948996 \\
\hline
\end{tabular}

number of the scenarios does not impact the robust optimal value. From Table 7, we can see that when the number of the scenarios increases, the optimal value has little change and no tendency to increase. We study in the other types of Solomon problems that the result of all problems shows the same trend. Therefore, determining the number of the scenarios is not an important factor affecting the quality of the experiment's results. From Table 8 , the ratio $P$ of performance for robust criteria clearly shows that the good decision criteria for this study are robust deviation and relative robustness. They provide the best performance for these situations.

\section{Conclusions}

In this study, we present a mathematical model of RVRPSTW to find the robust solution that provides the best of the worst case performance for VRPSTW under uncertain travel times. From the experiment, we found that the important parameter that affects the solutions is uncertainty percentage. The scenarios do not impact the robust optimal value. The good decision criteria for this study are robust deviation and relative robustness. The selection of different criteria creates different solutions which are appropriated depending on the purpose of the decision maker. The important thing for the decision maker is to determine the uncertainty percentage to cover all the uncertainties that need to be considered. In summary, the results in this paper can benefit the decision maker to make a decision for the same problem.

\section{Data Availability}

The data used to support the findings of this study have been deposited in Solomon's VRPTW.

\section{Conflicts of Interest}

The authors declare no conflicts of interest.

\section{Acknowledgments}

This research was supported by Chiang Mai University, Rajamangala University of Technology Isan, and the Centre of Excellence in Mathematics, CHE, Si Ayutthaya Rd., Bangkok, Thailand.

\section{References}

[1] G. B. Dantzig and J. H. Ramser, "The truck dispatching problem," Management Science, vol. 6, no. 1, pp. 80-91, 1959. 
[2] P. Kabcome and T. Mouktonglang, "Vehicle routing problem for multiple product types, compartments, and trips with soft time windows," International Journal of Mathematics and Mathematical Sciences, vol. 2015, Article ID 126754, 9 pages, 2015.

[3] É Taillard, P. Badeau, M. Gendreau, F. Guertin, and J.-Y. Potvin, "A tabu search heuristic for the vehicle routing problem with soft time windows," Transportation Science, vol. 31, no. 2, pp. 170-186, 1997.

[4] H. Min, "A multiobjective vehicle routing problem with soft time windows: the case of a public library distribution system," Socio-Economic Planning Sciences, vol. 25, no. 3, pp. 179-188, 1991.

[5] A. Agra, M. Christiansen, R. Figueiredo, L. M. Hvattum, M. Poss, and C. Requejo, "The robust vehicle routing problem with time windows," Computers \& Operations Research, vol. 40, no. 3, pp. 856-866, 2013.

[6] D. Tas, N. Dellaert, T. V. Woensel, and T. D Kok, "Vehicle routing problem with stochastic travel times including soft time windows and service costs," Computer and Operation Research, vol. 40, no. 1, pp. 214-224, 2013.

[7] M. M. Solomon, "Algorithms for the vehicle routing and scheduling problems with time window constraints," Operations Research, vol. 35, no. 2, pp. 254-265, 1987.

[8] G. B. Dantzig, "Linear programming under uncertainty," Management Science, vol. 50, no. 12, pp. 1764-1769, 2004.

[9] A. Charnes and W. W. Cooper, "Chance-constrained programming," Management Science, vol. 6, no. 1, pp. 73-79, 1959.

[10] A. Ben-Tal, L. El Ghaoui, and A. Nemirovski, Robust Optimization, Princeton University Press, Princeton, NJ, USA, 2009.

[11] P. Kouvelis and G. Yu, Robust Discrete Optimization and its Applications, Kluwer Academic Publishers, Dordrecht, Netherlands, 1997. 


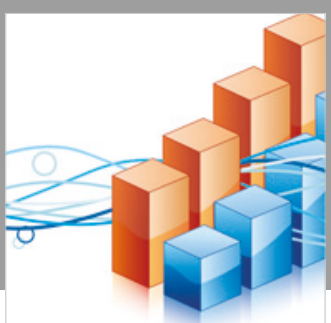

Advances in

Operations Research

\section{-n-m}
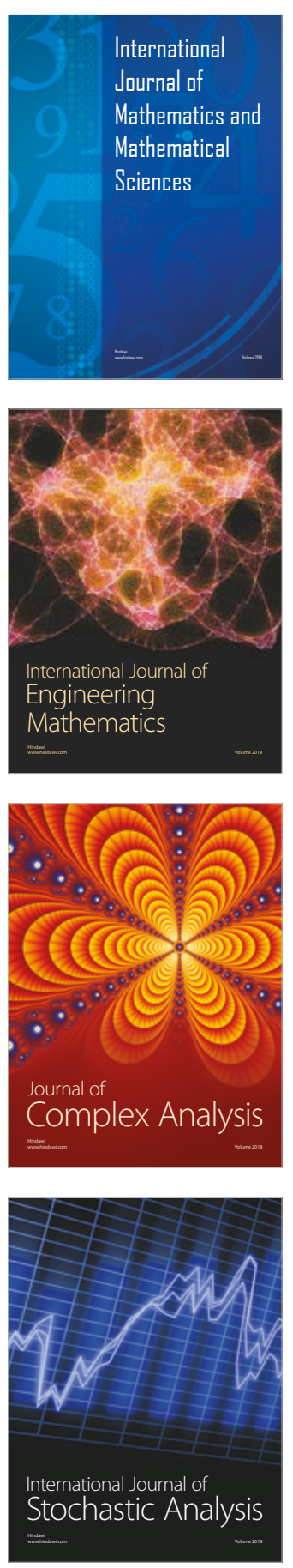
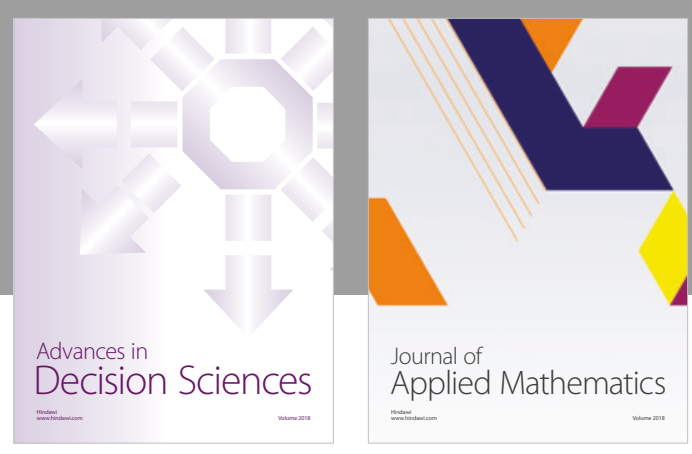

Journal of

Applied Mathematics
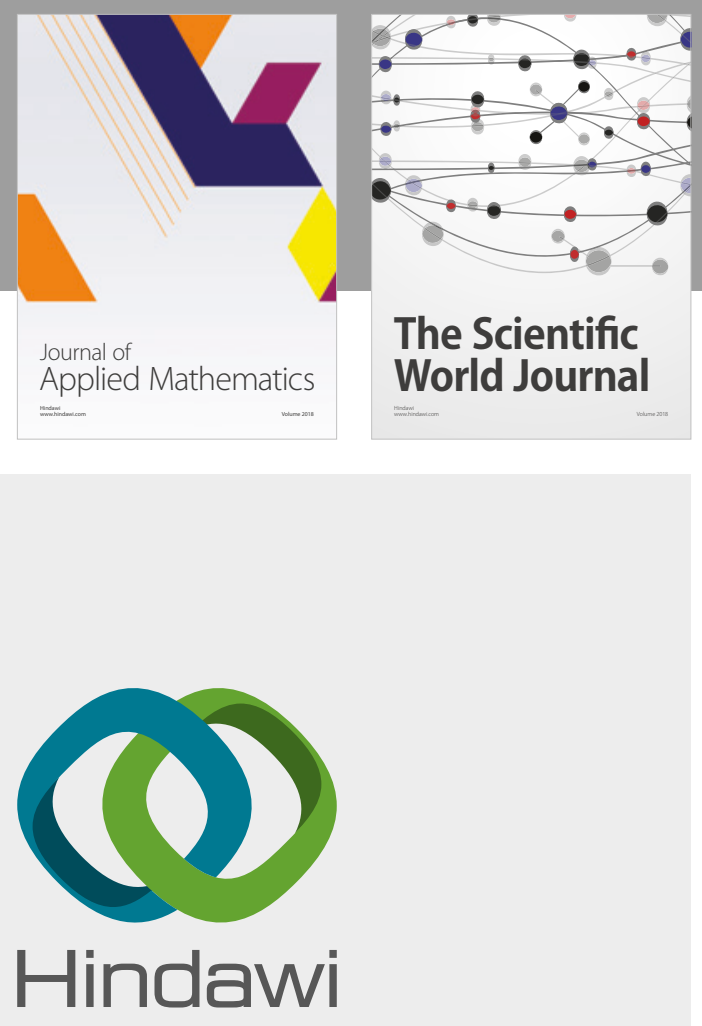

Submit your manuscripts at

www.hindawi.com

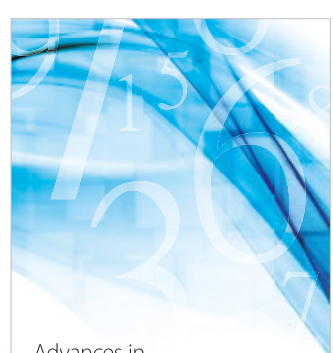

Advances in
Numerical Analysis
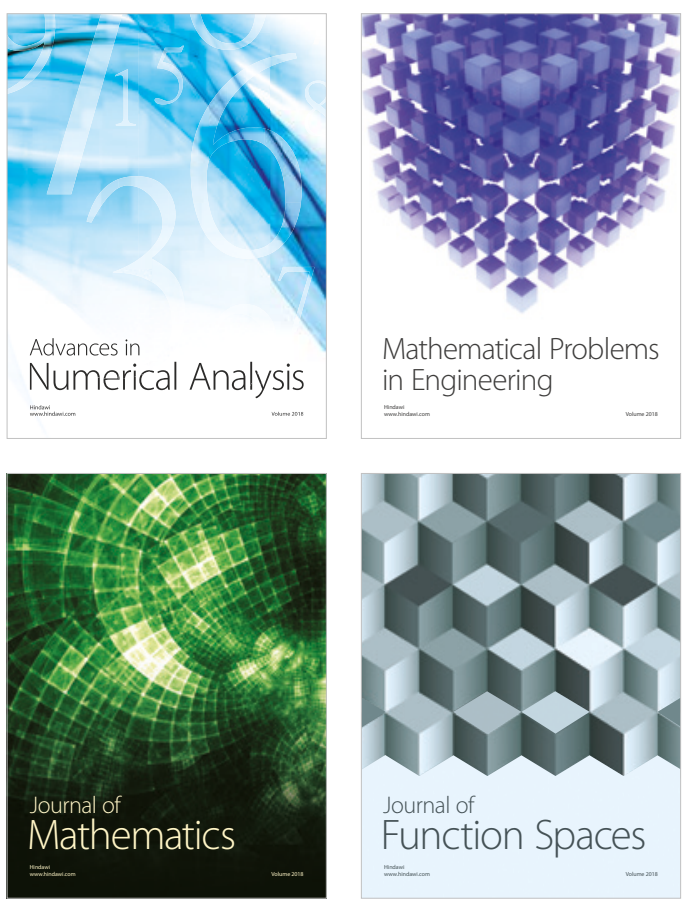

Mathematical Problems in Engineering

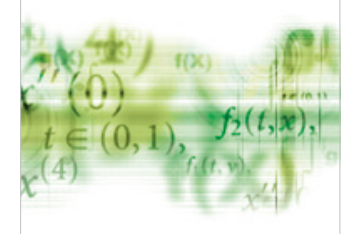

International Journal of

Differential Equations

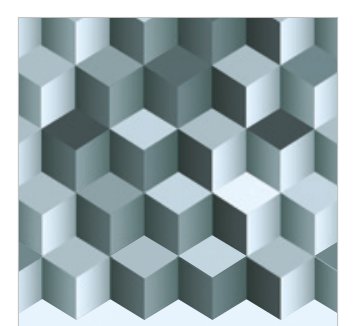

Journal of

Function Spaces
The Scientific

World Journal

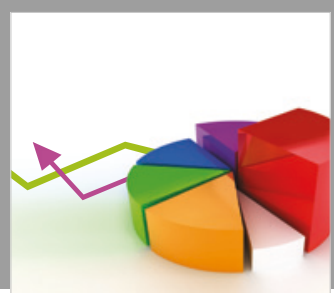

Journal of

Probability and Statistics
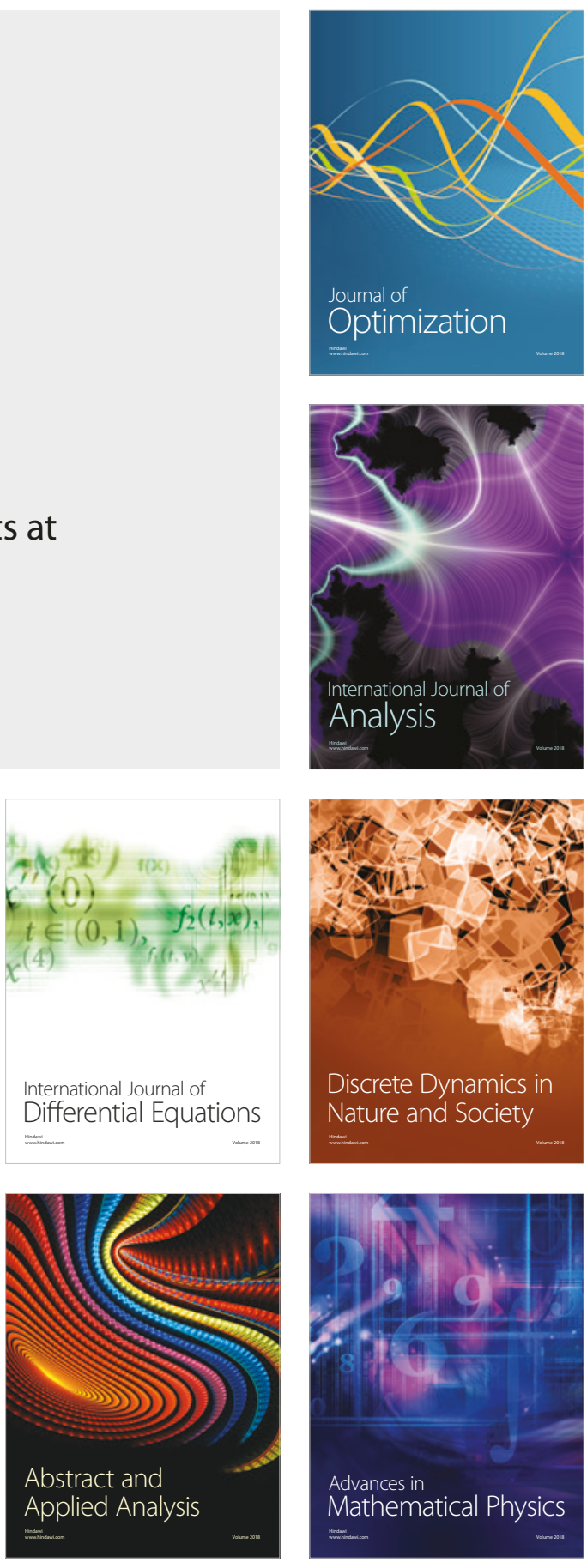